\title{
Pineal Parenchymal Tumor with Intermediate Differentiation-A Case Report and Review of Literature from Rural India
}

\author{
Raj Kumar $^{1}$ Seema Dayal ${ }^{2}$ Mani Krishna ${ }^{1}$ \\ ${ }^{1}$ Department of Neurosurgery, Uttar Pradesh University of Medical \\ Sciences, Saifai, Etawah, Uttar Pradesh, India \\ 2Department of Pathology, Uttar Pradesh University of Medical \\ Sciences, Saifai, Etawah, Uttar Pradesh, India
}

\begin{abstract}
Address for correspondence Seema Dayal, MD, Department of
Pathology, Uttar Pradesh University of Medical Sciences, Saifai,
Address for correspondence Seema Dayal, MD, Department of
Pathology, Uttar Pradesh University of Medical Sciences, Saifai, Etawah 206130, UP, India (e-mail: seemadayal5@gmail.com). Etawah 206130, UP, India (e-mail: seemadayal5@gmail.com).
\end{abstract}

Indian J Neurosurg 2019;8:55-57
Pineal gland tumors are rare tumors. According to World Health Organization (WHO) classification (2016) of the central nervous system, pineal parenchymal tumors of intermediate differentiation (PPTID) occupied their position between pineocytoma and pineoblastoma. It is either grade II or III. There are small numbers of reported cases, so its classification is still a matter of controversy. Here, we report a case of a 35-year-old man who presented with complaints of headache, nausea, vomiting, and focal weakness for 1 year. Computed tomography revealed an enhancing mass lesion in the region of pineal gland along with hydrocephalus. Following operation, histopathology of excised specimen was done, and it showed cellular tumor. Cells were having larger nuclei, vesicular chromatin pattern, ample cytoplasm, focal neuropil-like stroma along with moderate atypia, 3 to 5 mitotic figures per 10 hpf (high-power field), marked vascular proliferation, and pseudo rosette. Immunohistochemistry was found strongly positive for glial fibrillary acidic protein (GFAP) and neuron-specific enolase (NSE), variable for synaptophysin, and negative for neurofilament and epithelial membrane antigen (EMA). These findings confirmed the diagnosis. As this tumor is rare with uncertain behavior following available treatment, the case report of PPTID grade III large cell type may enrich the existing data and is being presented because of its rarity.

\section{Introduction}

Pineal tumors are rare, comprising 0.4 to $1 \%$ of brain tumors in adults and 3 to $8 \%$ in children. The major categories of pineal tumors include germ cell tumors, pineal parenchymal tumors (PPTs), and supporting tissue neoplasms (glial tumors). ${ }^{1}$ Germinomas are the most common histologic type, comprising 50 to $60 \%$ of all pineal tumors whereas PPTs aggregate about $30 \%$ of pineal region neoplasms. ${ }^{1,2}$ PPTs are subdivided into pineocytoma, pineoblastoma, and pineal parenchymal tumor of intermediate differentiation (PPTID). Pineoblastoma and pineocytoma aggregate about $45 \%$ each, and the remaining $10 \%$ are PPTID. ${ }^{3,4}$ PPTID was recognized in 2007 by the World Health Organization (WHO) as a new pineal parenchymal neoplasm intermediate in malignancy (WHO grades II and III) between pineocytoma and pineoblastoma. ${ }^{4}$

These tumors are composed of diffuse sheets or lobules of monomorphic, uniform, round cells with moderate to high cellularity, mild to moderate nuclear atypia, low to moderate mitotic activity along with vascular proliferation, giant cells, and Homer Wright rosettes. Despite that there is marked cellularity endothelial proliferation and necrosis is seen rarely, when present, it is accompanied with other degenerative changes and lacks peripheral nuclear palisading. Morphologically recognizable neuronal or ganglionic differentiation occurs rarely. ${ }^{5}$ In comparison with pineocytomas, they have higher potential for invasion, recurrence, and leptomeningeal dissemination.
DOI https://doi.org/

$10.1055 / \mathrm{s}-0039-1698846$ ISSN 2277-954X.
License terms

(이 (1) $\Theta \circledast$ 
Very few cases are reported of PPTID and even more rarely of grade III. Therefore, little is known about their clinical behavior and optimal management. Here, we present a case of PPTID grade III large cell type in a young adult.

\section{Case Report}

A 35-year-old man presented in neurosurgery OPD of Uttar Pradesh University of Medical Sciences, Saifai, Etawah, UP, India, with an intermittent headache, nausea, vomiting, and giddiness for 1 year. Computed tomographic (CT) findings revealed a pineal gland space occupying mass of about $4.5 \times$ $4 \mathrm{~cm}$ size. It was solid, heterogeneously enhancing mass with ill-defined margins. There was no evidence of calcification, but it was obstructing the posterior third ventricle, to cause gross hydrocephalous. The patient was further investigated to rule out secretory germ cell tumor. In view of hydrocephalous, a cerebrospinal fluid (CSF) diversion procedure (right ventriculoperitoneal shunt) was done, and the patient was taken for definitive surgery. At operation, tumor was grayish, vascular, and soft to firm at places. Decompression and biopsy were done, and the patient recovered following surgery. The received specimen was gray/brown soft tissue bits aggregating $1 / 2 \mathrm{cc}$, and it was sent in $10 \%$ formal saline in histopathology section of the pathology department. The tissue was processed, sectioned at 3 to $4 \mu \mathrm{m}$, and H\&E stain was applied.

Section screened showed round to oval cells arranged in diffuse flat sheets with blood vessels proliferation dominance. Cells were having larger nuclei, vesicular chromatin pattern, ample cytoplasm, and focal neuropil-like stroma along with moderate atypia, and mitotic activities were also appreciated, which was 3 to 5 mitotic figures per $10 \mathrm{hpf}$ (high-power field) ( - Fig. 1). All these findings were suggestive of large cell-type PPTID. Immunohistochemistry was found strongly positive for glial fibrillary acid protein (GFAP) and neuron-specific enolase (NSE), variable for synaptophysin, and

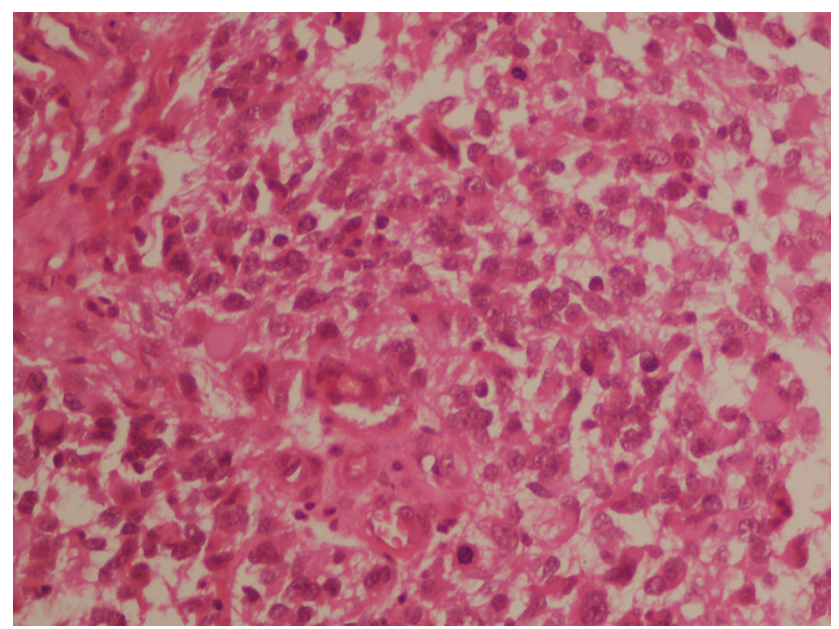

Fig. $1 \mathrm{H} \& \mathrm{E}(10 \times)$-stained section of pineal parenchymal tumor of intermediate differentiation showing singly arranged cells. Cells had larger nuclei, vesicular chromatin pattern, ample cytoplasm, and mitotic figures separated and supported by stromal elements with vascular proliferation.

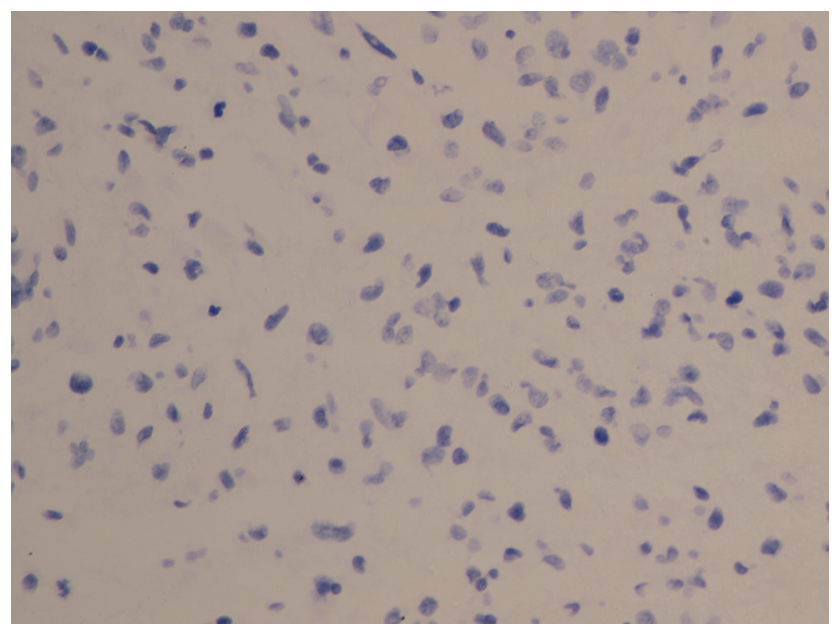

Fig. 2 Section showing negative immunostaining for neurofilament.

negative for epithelial membrane antigen (EMA) and neurofilament (-Fig. 2).

Based on its location, histomorphology, and immunohistochemistry analysis, it was diagnosed as PPTID grade III large cell type.

\section{Discussion}

The pineal gland is a small, red brown, pine-shaped endocrine gland located in the vicinity of the posterior third ventricle. Its size ranges between 10 and $14 \mathrm{~mm}$. Tumors of the pineal gland are classified as germ cell tumors, parenchymal tumors, and malformative tumors. The other pathologies include astrocytoma, meningiomas, ependymomas, metastatic brain tumors, and epidermoid and dermoid cyst. PPTIDs are the tumors that are intermediate in nature clinically and on microscopy. It constitutes approximately $10 \%$ of PPTs. Clinical profile of PPTID is similar to that of other pineal region tumors. Diplopia and headache are the common findings that are followed by Parinaud's syndrome. Parinaud's syndrome is vertical gaze disturbance that occurs due to compression of the tectal plate. If the tumor mass is large enough, it may cause hydrocephalous further leading to elevated intracranial pressure and ataxia. ${ }^{6}$ In one case series, it was found that $80 \%$ of PPTIDs have local invasion. ${ }^{7}$ Rarer complications include intracranial dissemination and CSF spread to the spine. The 5 -year survival rate is 39 to $74 \%{ }^{3}$ Tumor grade, extent of resection, and neuraxis spread were prognostic factors for freedom from progression and overall survival whereas high-grade histology and subtotal resection were the variables that negatively influenced an overall survival. Mallick et $\mathrm{al}^{8}$ found that the progression-free survival was 5.17 years and overall survival was 14 years. The possibilities of recurrence and spread are always there, including local, spinal, and leptomeningeal metastasis. Jouvet et $\mathrm{al}^{2}$ reported PPT with intermediate differentiation and graded it on the basis of mitosis and neurofilament staining. Patil and Karandikar $^{9}$ reported PPTID grade II along with metastasis in the thoracic and lumbosacral region. Khaled et $\mathrm{al}^{10}$ and Kiyici and Sanal ${ }^{11}$ also reported PPTID. Raleigh et $\mathrm{al}^{12}$ classified PPTIDs 
on their cell morphology and categorized them into small and large cell types, which had distinct clinical outcomes. Large cell types possibly have more adverse effects. The accurate frequency of occurrence is difficult to evaluate because of diagnostic difficulties both clinically and pathologically and also subjective to the pathologist. Imaging in these tumors is also supplementary for diagnosis. CT results vary from heterogenous to uniform enhancement whereas on magnetic resonance imaging, tumors are heterogenous hypointense on T1WI and heterogenous hyperintense on T2WI. Therefore, there is no single neuroimaging feature that is pathognomic of PPTID. ${ }^{13}$ This category was first introduced by Schild et al in $1993,{ }^{14}$ and this tumor occurs in all age groups from childhood to adult and the peak incidence early adults. ${ }^{5}$ This case was diagnosed in a 35 -year-old man. Jouvet et $\mathrm{al}^{2}$ proposed a new prognostic grading grade I pineocytoma, grade IV pineoblastoma, and grades II and III are for PPTID that lies between pineocytoma and pineoblastoma. The grade II have less than 6 mitotic figures per $10 \mathrm{hpf}$, with positive immune labeling for neurofilament and grade III being defined as having 6 or more mitotic figures per $10 \mathrm{hpf}$ or less than 6 mitotic figures but without immune staining for neurofilament. In PPTIDs, the expression of neuronal markers is variable. Staining for synaptophysin is mainly diffuse, cytoplasmic, and variable in intensity. Neurofilament protein expression is variable. GFAP and S-100 protein staining are also positive. Chromogranin A can be expressed with pseudo-stratified architecture. ${ }^{9,10,14}$ Immunohistostaining is not significant to differentiate small and large cell-type PPTIDs. ${ }^{12}$

We present the case report of large cell-type PPT grade III. This case should be discussed as it is valuable because accurate diagnosis is mandatory for the further management.

\section{Conclusion}

Diagnosing PPTID can be difficult, and histopathologic findings are not pathognomonic. Histopathology along with immune histochemistry must be done to diagnose and rule out differential. As accurate grading is essential for such rare tumors for their optimal therapeutic management and prognosis that depend on the histopathologic grading. CSF analysis and spinal screening with clinical follow-up should be done because the possibilities of dissemination. More study should be carried out on this tumor to better interpret the nature of PPTID, especially large cell types as they are associated with poor prognosis.

\section{Conflicts of Interest}

None declared.

\section{References}

1 Gasparetto EL, Warszawiak D, Adam GP, Bleggi-Torres LF, de Carvalho Neto A. Glioblastoma multiforme of the pineal region: case report. Arq Neuropsiquiatr 2003;61(2B):468-472

2 Jouvet A, Saint-Pierre G, Fauchon F, et al. Pineal parenchymal tumors: a correlation of histological features with prognosis in 66 cases. Brain Pathol 2000;10(1):49-60

3 Fauchon F, Jouvet A, Paquis P, et al. Parenchymal pineal tumors: a clinicopathological study of 76 cases. Int J Radiat Oncol Biol Phys 2000;46(4):959-968

4 Mena H, Nakazato Y, Jouvet A, Pineal parenchymal tumours. In: Kleiheus P, Cavenee WK, eds. The WHO Classification of Tumours of the Central Nervous System, Lyon, France: IARC Press; 2002:115-122

5 Nakazato Y, Jouvet A, Scheitauer BW, Pineal parenchymal tumor of intermediate differentiation. In: Louis DN, ed. World Health Organization Classification of Tumours of the Central Nervous System. 4th ed, Vol. 7. WHO; 2007:124-125

6 Amendola BE, Wolf A, Coy SR, Amendola MA, Eber D. Pineal tumors: analysis of treatment results in 20 patients. J Neurosurg 2005;102(Suppl):175-179

7 Komakula S, Warmuth-Metz M, Hildenbrand P, et al. Pineal parenchymal tumor of intermediate differentiation: imaging spectrum of an unusual tumor in 11 cases. Neuroradiology 2011;53(8):577-584

8 Mallick S, Benson R, Rath GK. Patterns of care and survival outcomes in patients with pineal parenchymal tumor of intermediate differentiation: an individual patient data analysis. Radiother Oncol 2016;121(2):204-208

9 Patil M, Karandikar M. Pineal parenchymal tumor of intermediate differentiation. Indian $\mathrm{J}$ Pathol Microbiol 2015;58(4):540-542

10 Khaled A, Joarder MA, Chandy M, Nasir TA. Pineal parenchymal tumour of intermediate differentiation: a case report. Pulse 2013;6(1-2):60-61

11 Kiyici H, Sanal B. Pineal parenchymal tumor of intermediate differentiation: a brief report. Eur J Basic Med Sci 2012;2(1):30-33

12 Raleigh DR, Solomon DA, Lloyd SA, et al. Histopathologic review of pineal parenchymal tumors identifies novel morphologic subtypes and prognostic factors for outcome. Neuro-oncol 2017;19(1):78-88

13 Amato-Watkins AC, Lammie A, Hayhurst C, Leach P. Pineal parenchymal tumours of intermediate differentiation-an evidence-based review of a new pathological entity. Br J Neurosurg 2016;30(1):11-15

14 Schild SE, Scheithauer BW, Schomberg PJ, et al. Pineal parenchymal tumors. Clinical, pathologic, and therapeutic aspects. Cancer 1993;72(3):870-880 